\title{
Mehr ist nicht immer ein Plus. Gemeinsam entscheiden!
}

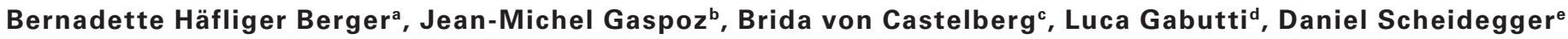 \\ ${ }^{a}$ Lic. iur., Rechtsanwältin, Geschäftsführerin smarter medicine, Generalsekretärin SGAIM; b Prof. Dr. med., Präsident smarter medicine, Co-Präsident SGAIM

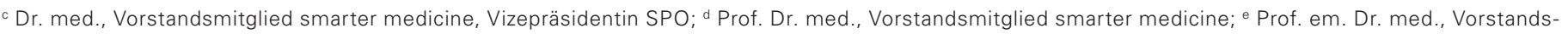 \\ mitglied smarter medicine, Präsident SAMW
}

\begin{abstract}
Der Verein smarter medicine - Choosing Wisely Switzerland hat eine Kampagne lanciert, welche das Thema der medizinischen Fehl- und Überversorgung der Öffentlichkeit verständlich machen will. Listen von Fachgesellschaften mit unnötigen Behandlungen wurden so umgeschrieben, dass sie auch medizinische Laien verstehen. Im Mittelpunkt steht aber das Gespräch zwischen Behandelnden und Behandelten.
\end{abstract}

Noch heute gilt, was der griechische Arzt und Philosoph Epiktet vor knapp 2000 Jahren postulierte: Einem Arzt, der nichts verschreibt, zürnen die Kranken und glauben, sie seien von ihm aufgegeben. Im modernen Gesundheitssystem kommt zudem die Befürchtung auf, dass medizinische Leistungen zu Lasten einzelner rationiert werden sollen. Gerade angesichts der rasanten Fortschritte der Medizin herrscht die Überzeugung vor, sämtliche verfügbaren Möglichkeiten müssten immer ausgeschöpft werden. Die Frage, ob diese im individuellen Fall überhaupt angezeigt sind und der Heilung bzw. der Verbesserung der Lebensqualität der Behandelten dienen, wird viel zu selten gestellt.

Die Initiative smarter medicine verfolgt den Grundsatz, dass eine Behandlung oder eine Diagnosemethode nicht angewendet wird, wenn sie den Betroffenen nichts nützen oder gar noch schaden. Der entsprechende Trägerverein wurde letztes Jahr gegründet und vereinigt medizinische Fach- und Berufsorganisationen mit Patienten- und Konsumentenorganisationen.

\section{Nur gut informierte und selbstbewusste} Patient/-innen sind in der Lage, Entscheidungen zu verstehen und mitzutragen.

smarter medicine lanciert nun eine zweijährige Kampagne unter dem Slogan «Mehr ist nicht immer ein Plus. Gemeinsam entscheiden", bei der die Sicht der Konsument/-innen und Patient/-innen ins Zentrum gerückt wird. Behandelte sollen das Gespräch um die richtige Diagnose- und Therapiemethode zusammen mit den
Behandelnden auf Augenhöhe führen können. Nur gut informierte und selbstbewusste Patient/-innen sind in der Lage, Entscheidungen zu verstehen und mitzutragen. Zudem müssen sie darauf vertrauen können, dass ihnen immer die für sie persönlich beste Behandlung zukommt. Das bedeutet aber nicht zwingend, dass alle zur Verfügung stehenden Mittel eingesetzt werden müssen.

\section{Ist Fehl- und Überversorgung überhaupt ein Problem?}

Studien zeigen, dass etwa 20 bis 30 Prozent der Gesundheitskosten durch Behandlungen verursacht werden, die medizinisch nicht angezeigt sind [1]. So steigt etwa die Zahl gelenkchirurgischer Eingriffe weit stärker, als dies epidemiologisch durch die Alterung zu erklären wäre [2]. Eine andere Studie belegt, dass in bestimmten Spitälern in der Schweiz bis zu 70 Prozent der elektiven Herzkatheteruntersuchungen vorgenommen werden, ohne dass pathologische Befunde vorliegen [3]. smarter medicine ist davon überzeugt, dass im schweizerischen Gesundheitssystem heute Anreize bestehen, die Patient/-innen kränker zu machen, als sie sind [4]. Ein Grund für die Überversorgung ist in der hohen Dichte an Spezialist/-innen resp. in der fortschreitenden Subspezialisierung zu suchen. Eine weitere Ursache dürfte in der fragmentierten und wenig koordinierten Versorgung zu finden sein. Das schweizerische Tarifsystem wirkt ebenfalls als Treiber hin zur Überversorgung, da sich Geld durch zusätzliche Untersuchungen und Behandlungen verdienen lässt. 


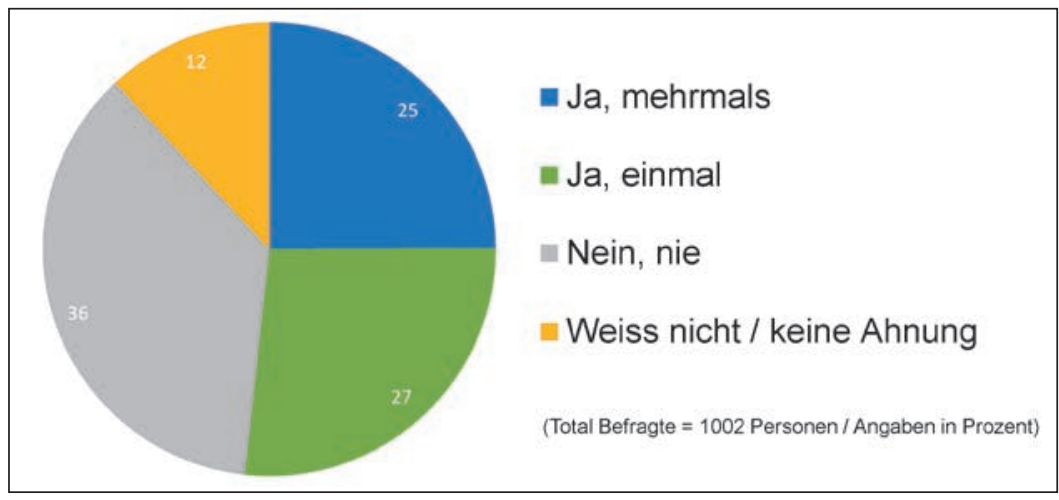

Abb. 1: GfK 2018 GfK eBus 2018 zur Frage: Hatten Sie oder jemand in Ihrem Umfeld schon jemals das Gefühl, eine Behandlung erhalten zu haben, die nicht nötig gewesen wäre?

Ein Beispiel dafür, dass einer solchen Entwicklung auch Gegensteuer gegeben werden kann, ist eine im Jahr 2014 lancierte Kampagne des Netzes der öffentlichen Tessiner Spitäler (Ente Ospedaliero Cantonale). Ausgangspunkt der interprofessionellen Intervention bildete, dass eine überdurchschnittlich hohe Verschreibung von Benzodiazepinen und Antazida (Protonenpumpenhemmer) festgestellt wurde, wobei eine relevante Variabilität zwischen den einzelnen Spitälern bestanden hat. Die Intervention basierte anfänglich auf einem Monitoring und dem Vergleich von Benchmark. Dann wurde das involvierte Personal direkt angesprochen und an Informations- und Fortbildungsveranstaltungen geschult. Dies erlaubte, die dauerhafte Verschreibung dieser beiden Medikamente wirkungsvoll zu reduzieren $[5,6]$.

\section{Was meinen die Patient/-innen?}

Im Zeitraum vom 28. Juni bis 3. Juli 2018 hat das Meinungsforschungsinstitut GfK im Auftrag des Träger-

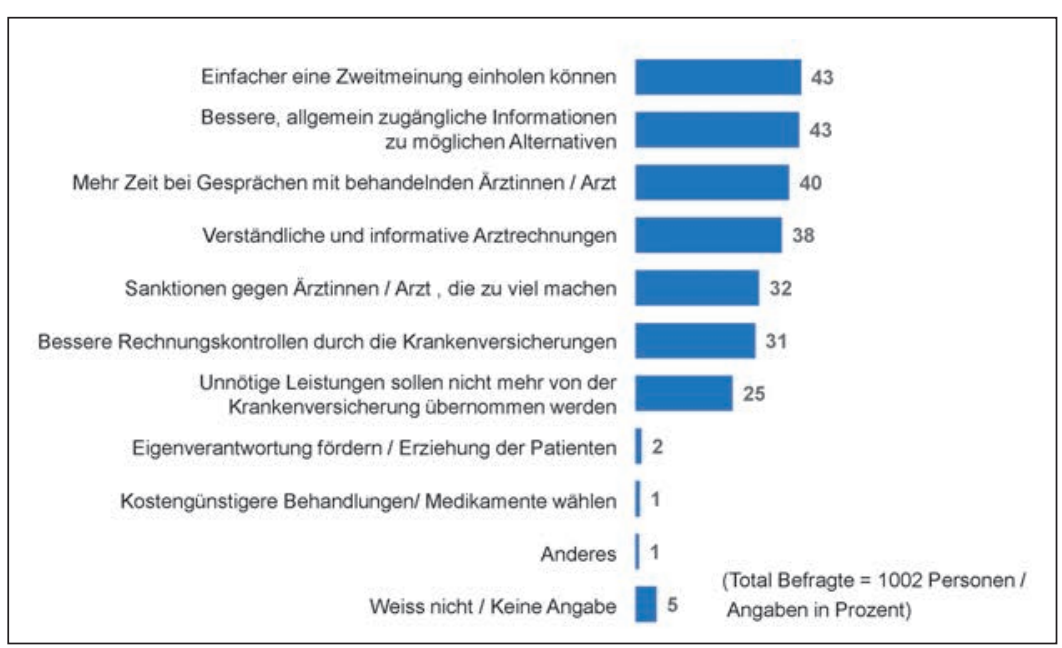

Abb. 2: GfK 2018 GfK eBus 2018 zur Frage: Was würde helfen, dass es nicht zu medizinischer Überversorgung kommen kann? Mit medizinischer Überversorgung meinen wir, es wird zu viel oder das Falsche gemacht. vereins eine Umfrage zur medizinischen Versorgung und zur Bekanntheit von smarter medicine durchgeführt. Über 1000 Antworten aus der Deutsch- und Westschweiz sorgen dafür, dass die Ergebnisse repräsentativ sind. Diese Umfrage soll am Schluss der Kampagne wiederholt werden, um die Wirkung der ergriffenen Massnahmen zu überprüfen. Wie vermutet kennen aktuell nur die wenigsten die Initiative (gerade einmal 6 Prozent).

Jedoch ist das angesprochene Problem der Überversorgung bekannt. Über die Hälfte der Befragten ist der Meinung, dass sie oder jemand aus ihrem Umfeld schon einmal oder gar mehrfach eine Behandlung erhalten hat, welche nicht nötig gewesen wäre. Umgekehrt sind 36 Prozent davon überzeugt, dass ihnen das noch nie passiert ist (Abb. 1).

Auf die Frage «Was würde helfen, dass es nicht zu medizinischer Überversorgung kommen kann?» nannten die Befragten als Hauptmassnahmen einerseits die einfachere Möglichkeit, eine Zweitmeinung einzuholen, und andererseits bessere, allgemein zugängliche Informationen zu möglichen Alternativen sowie mehr Zeit beim Gespräch mit der behandelnden Ärzteschaft. Die Eigenverantwortung der Patienten zu fördern, erachten jedoch nur 2 Prozent als adäquates Mittel gegen Überversorgung. Dagegen wünschen sich über 30 Prozent der Befragten Sanktionen gegen Ärztinnen und Ärzte, die zu viel machen (Abb. 2).

Die gesamten Umfrageergebnisse wurden unter www. smartermedicine.ch publiziert.

\section{Bewegung bei medizinischen Fachgesellschaften}

Eine zentrale Rolle bei smarter medicine nehmen medizinische Fachgesellschaften ein. Sie sind es, die für ihren Fachbereich Empfehlungen, die sogenannten «Top-5-Listen», erarbeiten und veröffentlichen. Die Top-5-Liste führt fünf Interventionen auf, die vermieden werden sollten. Die Fachgesellschaften liefern also das eigentliche Grundlagenmaterial. Bei der Gründung des Vereins smarter medicine im Sommer 2017 wurden alle Fachgesellschaften aufgefordert, Listen in ihren Bereichen zu erarbeiten und zu publizieren. In der Zwischenzeit sind sieben Fachgesellschaften dieser Aufforderung gefolgt. Weitere sind aktuell daran, solche Listen zu erarbeiten. Ziel ist jedoch, dass sich alle medizinischen Fachgesellschaften und auch andere nichtärztliche Organisationen der Initiative anschliessen. Jüngst haben beispielsweise verschiedene Spitäler aus den Kantonen Genf, Zürich und Tessin die Plattform «Smarter Hospital» gebildet [7]. 


\section{Spezifische Informationen und ein erster «Patiententag"}

Unterdessen wurden die bestehenden Empfehlungen in eine verständlichere und einfachere Sprache übersetzt. Auf dieser Basis können nun die Patientinnen und Patienten besser einbezogen werden. Die Informationen werden in erster Linie auf der Website www. smartermedicine.ch veröffentlicht und sind so breit zugänglich gemacht. Daneben werden Erklärvideos eingesetzt und eine Social-Media-Kampagne geführt, welche für das Thema der Fehl- und Überversorgung sensibilisieren möchte. Am 20. September 2019 soll dann der erste nationale Patiententag in St.Gallen stattfinden, den der Verein zusammen mit verschiedenen Organisationen aus dem Gesundheitsbereich organisieren wird.

\section{smarter medicine prangert auch die Verschwen- dung von Ressourcen im Gesundheitswesen an.}

Korrespondenz: smarter medicine -

Choosing Wisely Switzerland c/o SGAIM

Monbijoustrasse 43

Postfach

CH-3001 Bern

smartermedicine[at]

sgaim.ch
Neben den spezifischen Patienteninformationen werden auf Wunsch der Ärzteschaft und in Zusammenarbeit mit dem Swiss Medical Board die wichtigsten Empfehlungen der Fachgesellschaften auch mit zusätzlichem Informationsmaterial (z.B. Faktenboxen, Plakate) für Leistungserbringer ergänzt.

\section{Strategie auch gegen Verschwendung}

smarter medicine prangert auch die Verschwendung von Ressourcen im Gesundheitswesen an, da sie einer- seits zu einer Kostensteigerung führt und andererseits den Druck hin zur Rationierung in der Medizin erhöht. Doch der Verein will mit der Patientenkampagne nicht a priori Kosten sparen, sondern in erster Linie die Qualität in der Medizin verbessern. Es ist davon auszugehen, dass mit einer konsequenten Einhaltung der Empfehlungen auch die Gesundheitskosten sinken.

\section{Referenzen}

1 Gerber M, Kraft E, Bosshard C. Overuse - unnötige Behandlungen als Qualitätsproblem. Schweiz Ärzteztg. 2016;97(7):236-43.

2 Rosemann T, Neuner-Jehle S, Muheim L. Versorgungsforschung das richtige Mass an Medizin, zum richtigen Zeitpunkt für den richtigen Patienten. Therapeutische Umschau. 2017;74(1):827-31.

3 Interventional Cardiology in Switzerland - Annual Statistic Report. 2015. Swiss Working Group of Interventional Cardiology. http://www.ptca.ch/DOCS_PUBLIC/ptca_statistics_2015.pdf.

4 Anderegg S. «Es besteht der Anreiz, die Patienten kränker zu machen, als sie sind.» Tages-Anzeiger vom 6.6.2018.

5 Del Giorno R, Ceschi A, Pironi M, Zasa A, Greco A, Gabutti L. Multifaceted intervention to curb in-hospital over-prescription of proton pump inhibitors: A longitudinal multicenter quasi-experimental before-and-after study. Eur J Intern Med. 2018;50:52-9. Doi: 10.1016/j.ejim.2017.11.002.

6 Del Giorno R, Greco A, Zasa A, Clivio L, Pironi M, Ceschi A, Gabutti L. Combining prescription monitoring, benchmarking, and educational interventions to reduce benzodiazepine prescriptions among internal medicine inpatients; a multicenter before and after study in a network of Swiss Public Hospitals. Postgrad Med. 2018;2:1-10. Doi: 10.1080/00325481.2018.1504594.

7 Gaspoz J-M. Smarter Hospital - für eine Medizin mit Augenmass. PHC. 2018;18(14):241.

Bildnachweis

Abbildungen 1 und 2: $\odot$ verein smarter medicine 